\title{
Collapsed and adsorbed states of a directed polymer chain in two-dimensions.
}

\author{
Pramod K Mishra and Yashwant Singh \\ Department of Physics, Banaras Hindu University, \\ Varanasi 221 005, India
}

(November 15, 2018)

\begin{abstract}
A phase diagram for a surface interacting long flexible partially directed polymer chain in a two-dimensional poor solvent where the possibility of collapse in the bulk exists is determined using exact enumeration method. We used a model of self attracting self-avoiding walk and evaluated 30 steps in series. An intermediate phase in between the desorbed collapsed and adsorbed expanded phases having the conformation of a surface attached globule is found. The four phases, viz. ( $i)$ desorbed expanded, $(i i)$ desorbed collapsed, (iii) adsorbed expanded, $(i v)$ surface attached globule are found to meet at a multicritical point. These features are in agreement with those of an isotropic (or non directed) polymer chain.
\end{abstract}

61.30 Cz, 62.20 Di, 61.30Jf

Typeset using REVTEX 


\section{INTRODUCTION}

The subject of adsorption of a long flexible polymer chain immersed in a poor solvent on an impenetrable surface with an attractive short-range attraction has received considerable attention in recent years 1 페. This is because an attractive surface may lead to adsorptiondesorption transition from the state when the chain is mostly attached to the surface to the state of detachment when the temperature is increased. This behaviour finds applications in lubrication, adhesion, surface protection, etc.

It is known that when a chain interacts with a surface its conformational properties get modified in comparison with its bulk properties [1,2]. This is because of a subtle competition between the gain of internal energy and the corresponding loss of entropy due to the constraint imposed on the chain by the impenetrable surface. This competition leads to the possibility of co-existence of different regimes and multicritical behaviour.

The essential physics associated with the behaviour of a surface interacting polymer chain in a good solvent is derived from a model of self-avoiding walk (SAW) on a semi-infinite lattice. If the surface is attractive, it contributes an energy $\epsilon_{s}(<0)$ for each step of the walk on the surface. This leads to an increased probability characterized by the Boltzmann factor $\omega=\exp \left(-\epsilon_{s} / k_{\beta} T\right)$ of making a step along the surface, since for $\epsilon_{s}<0, \omega>1$ for any finite temperature $T$ ( $k_{\beta}$ is the Boltzmann constant). Because of this the polymer chain get adsorbed at low temperatures on the surface while at high temperatures all polymer conformations have almost same weight and non adsorbed (or desorbed) behaviour prevails. The transition between these two regimes is marked by a critical adsorption temperature $T_{a}$, with a desorbed phase for $T>T_{a}$ and adsorbed phase for $T<T_{a}$ as shown in Fig. 1. At $T<T_{a}$ the chain acquires a conformation such that a fraction of monomers get attached to the surface and others form a layer of finite thickness parallel to the surface. The thickness of the layer diverges at $T=T_{a}$. One may define the crossover exponent $\phi$, at $T=T_{a}$ as $N_{s} \sim N^{\phi}$ where $N$ is the total number of monomers and $N_{s}$ the number of monomers on the surface. The transition point $T_{a}$ is a tricritical point [2]. Both the surface and the bulk critical exponents 
have been calculated using renormalization group methods [5], transfer matrix methods [6,7], exact enumeration method [8] and Monte Carlo methods [9]. For a two dimensional system exact values of the exponents have been found by using conformal invariance [10].

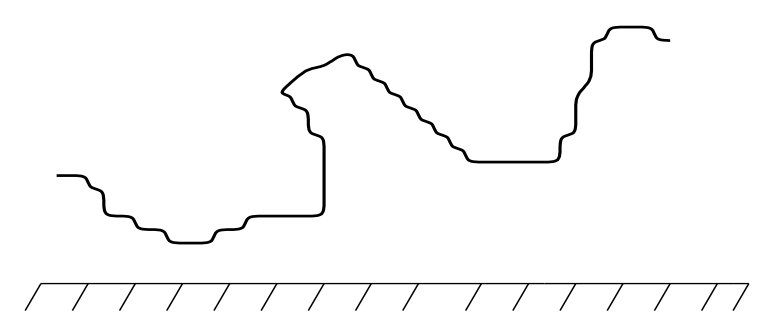

(a)

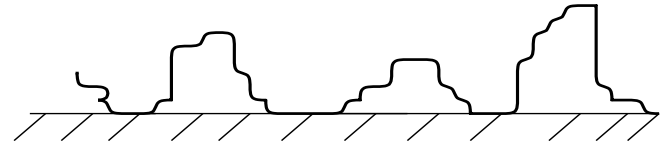

(b)

Fig. 1; Schematic representation of polymer conformations in (a) desorbed and (b) adsorbed states.

The situation is, however, different when the surface interacting polymer is a in compact globule state. In the vicinity of an impenetrable attracting surface the monomer-monomer attraction which is responsible for the collapsed state of the chain and the surface-monomer attraction responsible for the adsorption will compete. This competition may give rise new features in the conformational behaviour of the chain. For instance, a globule can get attached to the surface at some value of monomer-surface attraction without any significant modification in its structure as shown in Fig. 2. Such a phase has been shown to exist in between the bulk collapsed and adsorbed phases in both two and three dimensions for isotropic i.e. non-directed) polymer chain and is called surface attached globule (SAG) phase [11,12]. In an adsorbed phase the number of monomers on the surface are proportional to $N$ and the thickness of the layer formed by the chain parallel to the surface proportional to $N^{0}$. On the 
other hand the bulk globule has one correlation length (the radius of gyration) which scales with $N$ as $R \propto N^{1 / d}$ where $\mathrm{d}$ is the dimensionality of the space. In the SAG phase we have two correlation lengths, one parallel and another perpendicular to the surface. They may scale with $N$ as $R_{\|} \propto N^{x_{\|}}$and $R_{\perp} \propto N^{x_{\perp}}$ where $\frac{1}{d} \leq x_{\|} \leq \frac{1}{d-1}$ and $0<x_{\perp} \leq \frac{1}{d}$.

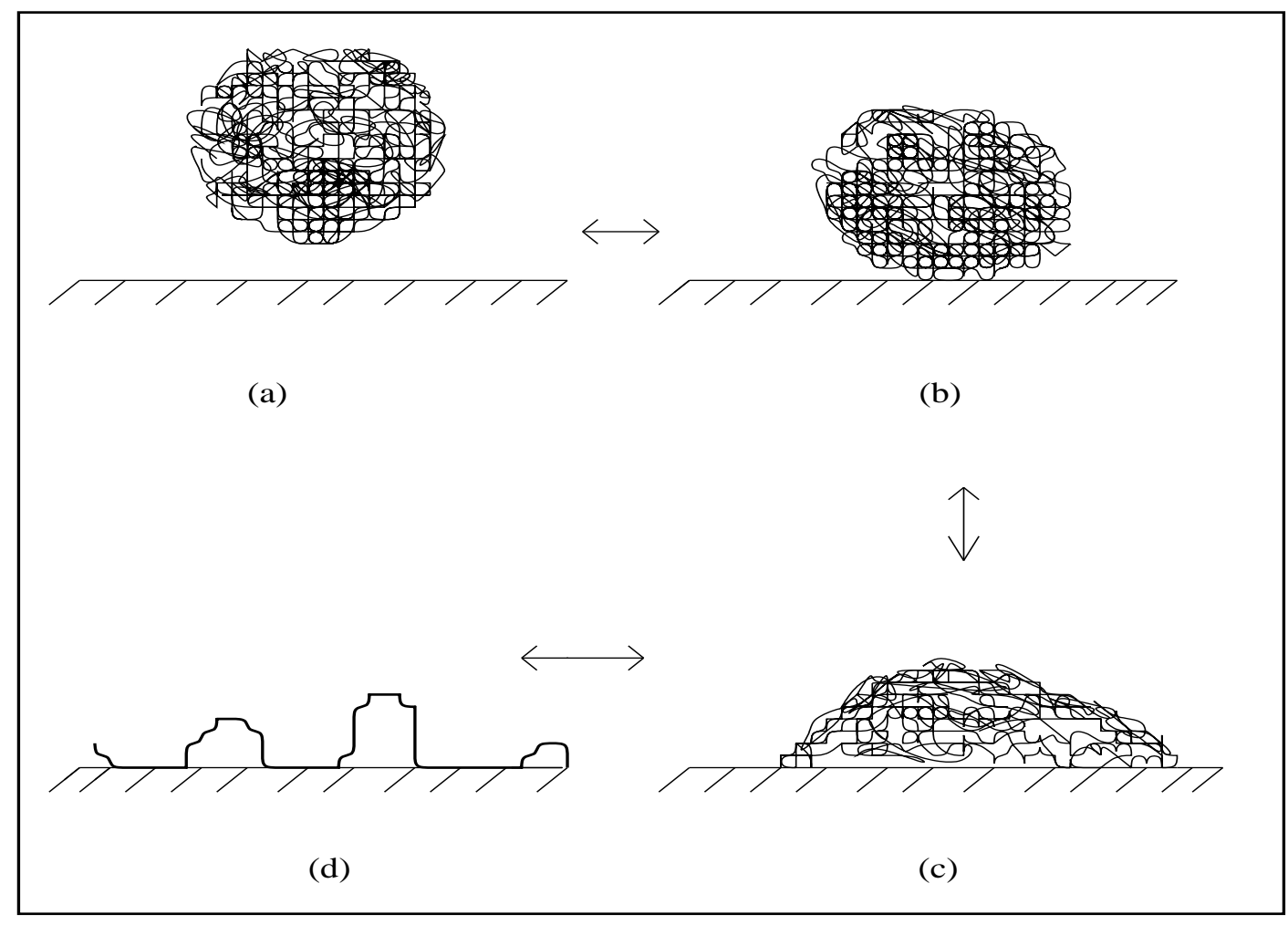

Fig. 2; Schematic representation of adsorption of a compact globule; (a) shows a desorbed globule, (b) and (c) surface attached globule and (d) the adsorbed state.

In this article we investigate the surface adsorption and collapsed of a directed polymer chain on a square lattice. Since for two dimensional system surface is a line, it can have only one adsorbed phase which we refer to as adsorbed expanded (AE) phase. The problem of adsorption and collapse of directed polymer has been studied using transfer matrix technique [13]. The phase boundary of the collapsed phase separating it from the adsorbed expanded and the bulk expanded has been obtained exactly [14] as well as numerically [15]. These cal- 
culations show that there are three phases, viz. desorbed expanded (DE), desorbed collapsed (DC) and adsorbed expanded (AE). These three phases meet at a multicritical point. In these calculations a condition $x u=1$ (where $x$ is the step fugacity and $u$ the Boltzmann factor for the monomer-monomer attraction) is used. This condition is valid only at the collapsed phase boundary. Therefore only the phase boundaries separating the DC phase from the AE and DE phases were obtained by these calculations. The phase boundary separating the DE phase from the AE phase has been obtained numerically by Veal et al 15. Since the transition from the DC phase to the SAG phase takes place in the region where $x u>1$ the calculations based on the transfer matrix could not show this transition.

\section{CALCULATIONAL DETAILS}

We consider partially directed self-attracting self-avoiding walks (PDSASAWs) on a square lattice restricted to quarter space. Walk starts from the corner of the surface. Let $C_{N, N_{s}, N_{p}}$ be the number of PDSAWs with $N$ steps, having $N_{s}(\leq N)$ steps on the surface and $N_{p}$ nearest neighbours. We have obtained $C_{N, N_{s}, N_{p}}$ for $N \leq 30$ for square lattice by exact enumeration method. We prefer this technique because in this case the scaling corrections are correctly taken into account by a suitable extrapolation scheme [9].

We associate energy $\epsilon_{s}$ with each monomer on the surface and $\epsilon_{p}$ for the monomer-monomer interaction. Partition function of the attached chain is

$$
Z_{N}(\omega, u)=\sum_{N_{s}, N_{p}} C_{N, N_{s}, N_{p}} \omega^{N_{s}} u^{N_{p}}
$$

where $\omega=e^{-\epsilon_{s} / k T}$ and $u=e^{-\epsilon_{p} / k T} \cdot \omega>1$ and $u>1$ for attractive force. Reduced free energy for the chain can be written as

$$
G(\omega, u)=\lim _{N \rightarrow \infty} \frac{1}{N} \log Z_{N}(\omega, u)
$$

In general it is appropriate to assume that as $N \rightarrow \infty$

$$
Z_{N}(\omega, u) \sim N^{\gamma-1} \mu(\omega, u)^{N}
$$


where $\mu(\omega, u)$ is the effective coordination number and $\gamma$ is the universal configurational exponents for walks with one end attached to the surface. The value of $\mu(\omega, u)$ can be estimated using ratio method [16] with associated Neville table or any other method such as Pade analysis [17] or differential approximants [18, 19].

From equations (2.2) and (2.3) we can write

$$
\log \mu(\omega, u)=\lim _{N \rightarrow \infty} \frac{1}{N} \log Z_{N}(\omega, u)=G(\omega, u)
$$

$Z_{N}(\omega, u)$ is calculated from the data of $C_{N, N_{s}, N_{p}}$ using equation (2.1) for a given $\omega$ and $u$. From this we construct linear and quadratic extrapolants of the ratio of $Z_{N}(\omega, u)$ for the adjacent values of $N$ as well as the alternate one. Results for alternate $N$ give better convergence. When $u=1$ and $\omega=1$ the value of $\mu$ is found to be 2.39 .

The surface critical exponent $\gamma_{1}$ can be calculated using the relation 8]

$$
\gamma^{0}-\gamma_{1}=\frac{\log \left(Z_{N}^{0} Z_{N-2} / Z_{N-2}^{0} Z_{N}\right)}{\log (N / N-2)}
$$

and the end-to-end distance exponent $\nu$ using the relation

$$
\nu=\frac{1}{2} \frac{\log \left(R_{N}^{2} / R_{N-2}^{2}\right)}{\log (N / N-2)}
$$

where the superscript " 0 " indicates that the quantities correspond to the bulk without the surface and $R_{N}$ is the end-to-end distance of a chain of $N$ monomers.

The value of $\omega_{c}(u)$ at which polymer gets adsorbed/attached to the surface for a given value of $u$ is found from the $(i)$ plot of $G_{s}(\omega, u)=G(\omega, u)-G(u, \omega=1)$ which remains equal to zero until $\omega=\omega_{c}$ and increases consistently as a function of $\omega$ for $\omega \geq \omega_{c}$, (ii) from the plot of $\partial^{2} G_{s}(\omega, u) / \partial \epsilon_{s}^{2}$ at constant $u$ and (iii) from the plot of $\gamma^{0}-\gamma_{1}$ as a function of $\omega$ for different $N$. The value of $\omega_{c}$ found from the plot of $G_{s}(\omega, u)$ is slightly lower than the peak value of $\partial^{2} G_{s} / \partial \epsilon_{s}{ }^{2}$. It is, however, observed that as $N$ is increased from 22 to 30 the peak value shifts to smaller $\omega$ and appears to converge on the value of $\omega$ found from $G_{s}(\omega, u)$ plot. We therefore choose the value of $\omega_{c}$ found from the plot of $G_{s}(\omega, u)$ and determine lines $\omega_{c}(u)$ and $\omega_{c 1}(u)$ (see Fig. 3 ) by this method. For $u=1$, the value of $\omega_{c}$ is $=1.70$ which is in very 
good agreement with the value 1.707 reported in ref. [6]. In another approach equation (2.5) is used to calculate $\gamma^{0}-\gamma_{1}$ for different $N$ and the values are plotted as a function of $\omega$. The value of $\omega_{c}$ is found from the intersection of successive approximation to $\gamma^{0}-\gamma_{1}$ in the limit $N \rightarrow \infty$. This method, however, fails for $u$ values above the $\theta$-point, $i$. $e$. where the desorbed phase is in a collapsed globule state. Below the $\theta$-point both the methods yield same values of $\omega_{c}$ which are in very good agreement.

The phase boundary separating the expanded and collapsed phases is calculated from the plot of $G_{b}(\omega, u)=G(\omega, u)-G(\omega, u=1)$ as a function of $u$ for a given $\omega$. Here $G_{b}$ measures the energy arising due to monomer-monomer attractions. This is however, not zero in the expanded phase and, therefore, cannot be used as an order parameter for the expanded and collapsed transition. We, however, find that the value of $G_{b}(\omega, u)$ as a function of $u$ shows sudden rise at certain value of $u$. We locate this point from the peak of $\partial^{2} G_{b} / \partial \epsilon_{p}^{2}$ which corresponds to a specific heat peak and shows divergence as $N \rightarrow \infty$. For $\omega=1$, the value of $u_{c}$ is 3.30 which is in good agreement with the value 3.38 found by exact calculation. The method is found to work for all values of $\omega$ i.e in both the bulk and the adsorbed regimes. However, as $\omega$ is increased the values of $G_{b}(\omega, u)$ do not remain as smooth as at lower values of $\omega$, therefore introducing some inaccuracy in the values of $u_{c}$. The values of $\left(\omega_{c}, u_{c}\right)$ at the multicritical point found by us are $(2.16,3.30)$ which are in good agreement with exactly known values $(2.19,3.38)$. As $\omega$ and $u$ becomes larger, higher order terms in equation (2.1) become significant and therefore to have proper convergence more terms must be considered. This explains the difference in $\omega_{c 2}$ line found by exact calculation 14 and the present calculation (see fig. 3).

\section{RESULTS}

The phase diagram shown in Fig. 3 has four phase boundaries instead of three as reported in earlier work [14,15]. The line $u_{c}$ separates the expanded and collapsed phases. 


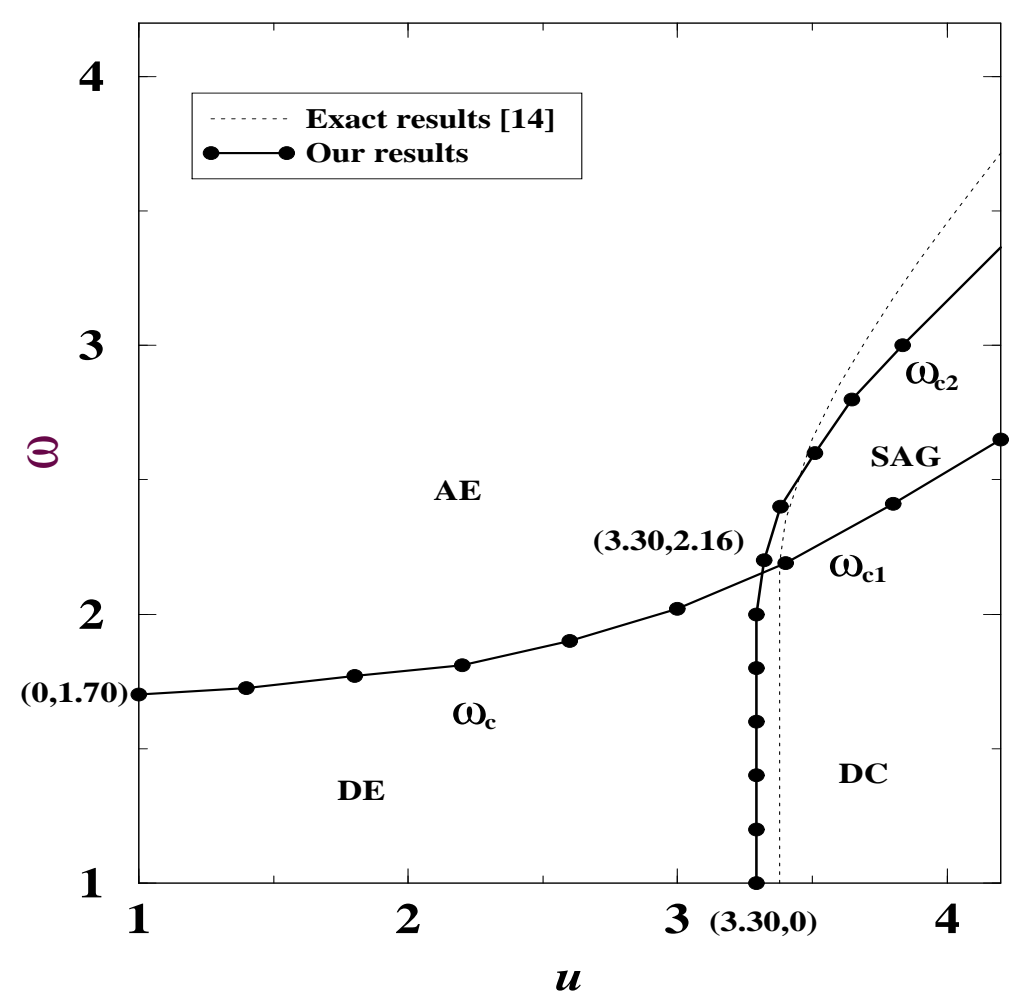

Fig. 3; Phase digram of a directed linear polymer chain in 2-D space. The $\omega$ and $u$ axes represents, the Boltzmann factor of surface attraction and monomer-monomer attraction respectively. Solid line curve corresponds to present calculation and the dotted line to ref. [14.

The special adsorption line $\omega_{c}$ separates adsorbed expanded (AE) phase from that of desorbed expanded (DE). Beyond $\theta$-point we have two boundaries $\omega_{c 1}$ and $\omega_{c 2}$. The line $\omega_{c 1}$ separates the desorbed collapsed (DC) bulk phase from that of a surface attached globule (SAG) state, whereas the boundary $\omega_{c 2}$ separates the SAG phase from the AE phase. The point where $u_{c}$ line meets the special adsorption line $\omega_{c}$, all four phases AE, DE, DC and SAG coexist. The SAG phase which exists between the boundaries $\omega_{c 1}$ and $\omega_{c 2}$ for $u>u_{c}$ is essentially a two-dimensional globule sticking to the surface in the same way as a liquid droplet may lie on a partially wet surface. These features are in agreement with those of isotropic (non directed) polymer chain. The average fraction of monomer on the surface and the average number of pairs are calculated using the relations.

$$
<n_{s}>=\left.\lim _{N \rightarrow \infty} w \frac{\partial G}{\partial \omega}\right|_{u}, \quad \text { and }<n_{p}>=\left.\lim _{N \rightarrow \infty} u \frac{\partial G}{\partial u}\right|_{\omega}
$$


The results are shown in Figs. 4 and 5. The transition points on each curve is marked by dots. The dot on a curve of $\left\langle n_{s}>\right.$ (in Fig. 4) indicates adsorption or polymer chain getting attached to the surface (i.e $\omega_{c}$ and $\omega_{c 1}$ lines depending on the values of $u$ ), the dot on a curve $<n_{p}>$ (in Fig. 5) indicates the collapsed-expanded states transition ( $i$. e. point on line $u_{c}$ and $\omega_{c 2}$ depending on the values of $\left.\omega\right)$. We may note that the value of $<n_{p}>$ for SAG phase is comparable to that in the DC phase.

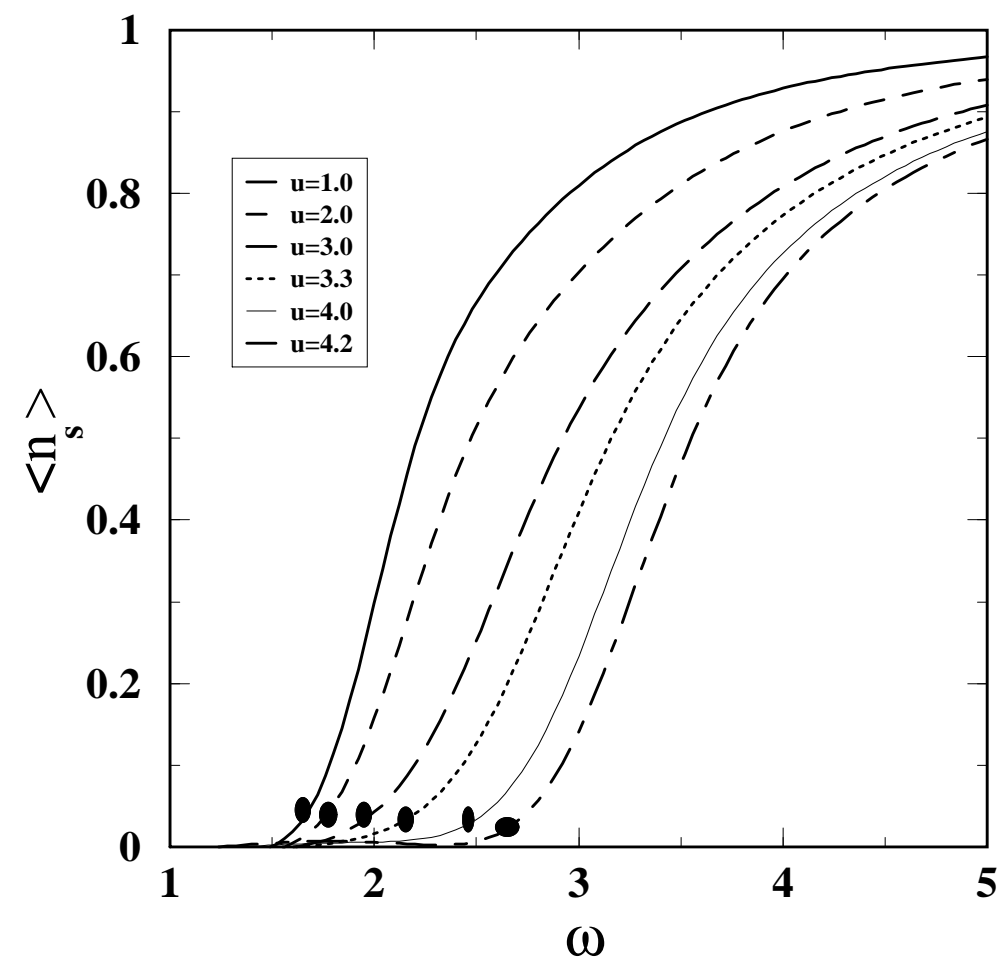

Fig.4; Average fraction of monomers on the surface $<n_{s}>$ as a function of $\omega$. Dot on the curve represents transition point.

We have found that along the line $\omega_{c}$ and $\omega_{c 1},<n_{s}>=0.04 \pm 0.01$ and along the line $u_{c}$ and $\omega_{c 2},<n_{p}>=0.54 \pm 0.06$ 


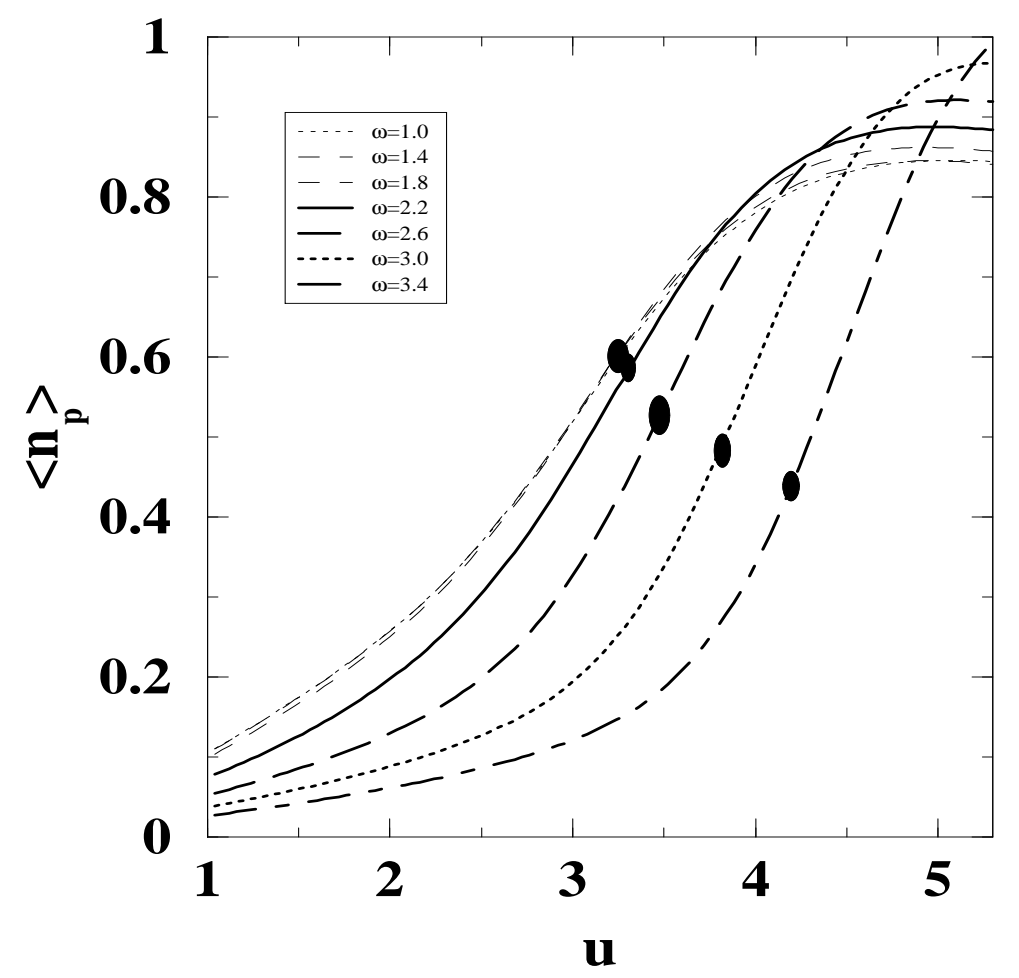

Fig.5; Average number of pairs $\left\langle n_{p}>\right.$ as a function of $u$. Dot on the curve represents the transition point.

\section{CONCLUSIONS}

It is obvious from these results that when the chain gets adsorbed from the expanded bulk state (i.e for $u<u_{c}$ ), it acquires a conformation at $\omega \simeq \omega_{c}(u)$ such that a small fraction of monomers get attached to the surface and others forms a layer parallel to the surface. This affects the free energy of the system. At the transition point the polymer chain fluctuates between adsorbed and desorbed conformations. Therefore one may get peak in $<N_{s}^{2}>-<N_{s}>^{2}$ at $\omega=\omega_{c}$ which shows divergence as $N \rightarrow \infty$. As $\omega$ is increased for the same value of $u$, the fluctuations along the normal to the surface get suppressed and at large $\omega\left(>>\omega_{c}\right)$ the chain lies on the surface with very little fluctuations. On the other hand, when the adsorbing chain was in collapsed bulk state, then at $\omega=\omega_{c 1}$ the collapsed chain gets attached to the surface. Here again the number of monomers getting attached to the surface 
are small. Since for $\omega \leq \omega_{c 1}$ the globule may be any where in the available space and get attached to the surface at $\omega=\omega_{c 1}$, there is a breaking of translational invariance at $\omega=\omega_{c 1}$ For $\omega_{c 1} \leq \omega \leq \omega_{c 2}(u)$ the chain remains in the form of a globule attached to the surface. In this range the monomer-monomer attraction remains effective in holding the monomers in the neighbourhood of each other than the surface-monomer attraction whose tendency is to spread the chain on the surface. Since at $\omega=\omega_{c 1}$ the globule retains its shape there is no change in the bulk free energy. Only the surface contribution to the free energy which was zero for $\omega \leq \omega_{c 1}$ starts contributing. Therefore, in this case we may therefore not see divergence in the bulk specific heat peak in the $N \rightarrow \infty$ limit. For $\omega>\omega_{c 2}(u)$ the globule conformation becomes unstable as surface-monomer attraction becomes more effective than the monomer-monomer attraction and therefore the chain spreads over the surface (just like a liquid droplet spreads over a wetting surface).

Summarizing we studied a polymer chain immersed in a poor solvent in the presence of an attracting impenetrable wall and obtained the phase boundaries separating different phases of the polymer chain from data obtained by exact enumerations. We report a state in between the desorbed collapsed and adsorbed phases which has conformation of a compact globule sticking to a surface in same way as a droplet may lie on a partially wet surface. A similar phase also been found in case of isotropic (non directed) polymer chain in both two and three dimensions [11, 12]. The number of monomers on the surface and the number of nearest neighbors in different regimes of the phase diagram are obtained.

\section{ACKNOWLEDGEMENTS}

We are thankful to Dr. S. Kumar and Dr. D. Giri for many fruitful discussions. The work was supported by the Department of Science and Technology (India) through project grant. 


\section{REFERENCES}

[1] E. Eisenriegler, Polymers Near Surfaces (World Scientific, Singapore) (1993).

[2] K. Dé Bell and T. Lookman, Rev. Mod. Phys. 65, 87 (1993).

[3] T. Vrbová and S. G. Whittington, J. Phys. A: Math Gen. 31, 3989 (1998).

[4] Y. Singh, S. Kumar and D. Giri J. Phys. A: Math. Gen. 25, L1211(1992)

[5] B. Derrida, J. Phys. A Math. Gen. 14, L5-L9 (1981); K. Kremer, J. Phys. A, 16, 4333 (1983); H. W. Diehl, Phase Transitions and Critical Phenomena, Vol. 10 edited by C. Domb and J. Lebowitz (Academic, New York), 76 (1986); E. Bouchaud E and J. Vannimenus, J. Physique 50, 2931 (1989).

[6] V. Privman, G. Forgacs and H. L. Frisch, Phys. Rev. B 379897 (1988); V. Privman and H. L. Frisch J. Chem. Phys. 88, 469 (1988).

[7] V. Privman and N. M. Svrakic, Directed Models of Polymers, Interfaces, and Clusters: Scaling and Finite-Size Properties. (Springer, Berlin, 1989); G. Forgacs, V. Privman and H. L. Frisch J. Chem. Phys. 90, 3339 (1989).

[8] D. P. Foster, E. Orlandini and M. C. Tesi, J. Phys. A:Math. Gen. 25, L1211 (1992).

[9] P. Grassberger and R. Hegger, Phys. Rev. E 51, 2674 (1995); P. Grassberger and R. Hegger, J. Phys. I. France 5, 597 (1995).

[10] M. T. Batchelor and C. M. Yung, Phys. Rev. Lett. 74, 2026 (1995).

[11] Y. Singh, D. Giri and S. Kumar, (cond-mat/0005493).

[12] Y. Singh, S. Kumar and D. Giri, (to be published).

[13] P-M Binder, A. L. Owczarek, A. R. Veal and J. M. Yeomans, J. Phys. A Math. Gen. 23, L975-L979 (1990).

[14] D. P. Foster and J. Yeomans, Physica A 177443 (1991); F. Igloi, Phys. Rev. A 43, 3194 
(1991).

[15] A. R. Veal, J. M. Yeomans and G. Jug, J. Phys. A: Math. Gen. 23, L109-L115(1990).

[16] D. S. Gaunt and A. J. Guttmann, Phase Transitions and Critical Phenomena, edited by C. Domb and M. S. Green (Academic, London), Vol. 3 (1974).

[17] D. L. Hunter and G. A. Baker, Phys. Rev. B 7, 3346 (1975).

[18] D. L. Hunter and G. A. Baker, Phys. Rev. B 19, 3808 (1979).

[19] A. J. Guttmann, Phase Transition and Critical Phenomena, edited by C. Domb and J. L. Lebowitz (Academic, New York), Vol 13 (1989). 